\title{
Embolia Pulmonar por Líquido Amniótico. Relato de Caso e Revisão da Literatura*
}

\author{
Pulmonary Amniotic Fluid Embolism Syndrome. \\ Case Report and Literature Review
}

\author{
Edmilton Pereira de Almeida ${ }^{1}$, Maria Antônia Campos Almeida², Lígia Menezes do Amaral ${ }^{3}$, \\ Cleber Soares Júnior ${ }^{4}$, Márcia Abudd Haddad ${ }^{5}$, Lúcia Lopes Rodrigues ${ }^{6}$
}

\section{RESUMO}

JUSTIFICATIVA E OBJETIVOS: A embolia pulmonar por líquido amniótico é uma complicação obstétrica rara, freqüentemente fatal, de fisiopatologia ainda não totalmente esclarecida e pouco conhecida pelo intensivista e obstetra. O objetivo deste relato foi enfatizar a necessidade de conhecimento desta entidade clínica e discutir a literatura pertinente de maior relevância.

RELATO DO CASO: Paciente com 15 anos, primigesta, 34 semanas de gestação, foi admitida no serviço de emergência para tratamento de início de parto prematu-

1. Especialista em Medicina Intensiva pela AMIB; Coordenador e Chefe da UTI Adulto do Hospital Monte Sinai; Professor Assistente de Cirurgia da UFJF; Especialista em Cirurgia Torácica pela SBPT-SBCT; Especialista em Nutrição Enteral e Parenteral pela SBNPE; Coordenador da Pós-Graduação em Terapia Intensiva da Faculdade de Ciências Médicas e da Saúde FCMSJF (SUPREMA), AMIB/SOMITI.

2. Especialista em Medicina Intensiva pela AMIB; Hematologista; Mestre em Hematologia pela UNIFESP.

3. Especialista em Medicina Intensiva pela AMIB; Pneumologista Titulada pela SBPT.

4. Especialista em Medicina Intensiva pela AMIB; Especialista em Cirurgia Gastroenterológica; Titular do CBC; Coordenador do Programa de Transplantes do Hospital Monte Sinai; Coordenador da Unidade Intermediária cirúrgica do HU-UFJF; Especialista em Nutrição Enteral e Parenteral pela SBNPE.

5. Especialista em Medicina Intensiva pela AMIB; Pneumologista.

6. Especialista em Medicina Intensiva pela AMIB

${ }^{*}$ Recebido do Hospital Monte Sinai, Juiz de Fora, MG.

Apresentado em 08 de dezembro de 2006

Aceito para publicação em 26 de abril de 2007

Endereço para correspondência:

Dr. Edmilton Pereira de Almeida

R: Renato Lage Mascarenhas, 55 - Vale do Ypê

36035-370 Juiz de Fora, MG

Fones: (32) 3213- 5372; 3215-5656; 3239-4455 r. 4130

Fax: (32) 3215- 5656.

E-mail: edmilton@terra.com.br

(C)Associação de Medicina Intensiva Brasileira, 2007 ro. Queixava-se de tosse e dispnéia. Ao exame clínico apresentava-se taquipneica (28 irm), ausculta pulmonar normal, sem outras anormalidades. Permaneceu em observação com uso de oxigênio através de máscara facial. Houve agravamento progressivo sendo transferida para a UTI. À admissão na UTI apresentava taquipnéia (28 irm), lúcida, pressão arterial (PA) de 104 x 56 mmHg e pressão arterial média (PAM) de $65 \mathrm{mmHg}, \mathrm{SpO}_{2}$ de $87 \%$ com fluxo de oxigênio de $5 \mathrm{~L} / \mathrm{min}$. Devido à piora progressiva da mecânica ventilatória foi realizada intubação traqueal e instituída ventilação mecânica com pressão controlada e volume garantido. Radiograma de tórax evidenciou infiltrado pulmonar extenso e bilateral. O painel de coagulação evidenciou importante alargamento do tempo de tromboplastina parcial ativado de 34,7 seg na admissão para 57,4 seg, 10 horas após, com redução progressiva nos dias subsequentes. Foi submetida à cesariana na manhã seguinte. Apresentou importante hipotensão arterial no intra-operatório, apesar de utilização de cristalóides em volume aparentemente adequado, sendo iniciado administração de noradrenalina. Foi extubada no $3^{\circ}$ dia, recebendo alta hospitalar 13 dias após a admissão, assintomática.

CONCLUSÕES: A embolia por líquido amniótico é uma rara e catastrófica complicação da gestação. A síndrome se caracteriza por um quadro súbito de desconforto ventilatório, hipotensão arterial, sintomatologia neurológica e coagulopatia que se iniciou durante ou em 30 min do trabalho de parto. O diagnóstico é de exclusão de outras afecções mais comuns, tais como eclâmpsia, sepse, miocardiopatia periparto, anafilaxia, tromboembolismo pulmonar, reações transfusionais, complicações anestésicas e estenose mitral, que podem surgir ou agravar-se durante o trabalho de parto. Pode ocorrer durante ou logo após aborto cirúrgico, amniocentese ou colocação de transdutor de pressão na cavidade uterina. $O$ tratamento é o de suporte hemodinâmico, ventilatório e correção dos distúrbios de 
coagulação. A interrupção imediata da gestação, através de cesariana, parece trazer benefícios para a mãe e concepto. Os intensivistas e obstetras devem estar atentos para esta entidade com o intuito de diagnóstico e tratamento imediatos.

Unitermos: embolia, gestação, líquido amniótico.

\section{SUMMARY}

BACKGROUND AND OBJECTIVES: Amniotic fluid embolism is a rare complication of pregnancy whose physiopathology is not completely known and still not frequently remembered by intensive care physicians and obstetricians. The main aim of this case report was to emphasize the need of better knowledge of this disease by physicians.

CASE REPORT: A 15 years old, primigravida, $35^{\text {th }}$ week of gestation was admitted in our intensive care unit (ICU) with a sudden respiratory distress. Soon after admission she was intubated and put on mechanical ventilation. After stabilization she was transferred to the operating room and a cesarean section was undertaken. During the operation, instead of fluid reposition with crystalloids in large amounts, her hemodynamic status deteriorated and it was necessary to use vasoactive drugs. After three days on mechanical ventilation she was successfully weaned and discharged from the intensive care unit (ICU) after 6 days. She and her baby were discharged from hospital on $13^{\text {th }}$ day of hospitalization.

CONCLUSIONS: Amniotic fluid embolism is a rare and catastrophic complication of pregnancy, at least in its classic presentation. It is characterized by acute respiratory distress, hemodynamic compromise and coagulopathy that occur during or within $30 \mathrm{~min}$ after labor. Diagnosis is by exclusion of other conditions of pregnancy or diseases that can be aggravated during pregnancy such as eclampsia, sepsis, pos-partum cardiomyopathy, anaphylaxis, pulmonary thromboembolism, transfusion reactions, anesthetic complications and mitral stenosis. There is growing evidence that we have a spectrum of manifestation which can be more common that in the classic ones. The treatment is supportive of vital functions, such as mechanical ventilation, fluid reposition, vasoactive drugs and fresh frozen plasma as necessary. The intensive care physicians and obstetricians should be aware of this disease in order to make early diagnosis and prompt treatment.

Key Words: amniotic fluid, embolism, pregnancy

\section{INTRODUÇÃO}

A embolia pulmonar por líquido amniótico é rara, mas muitas vezes fatal complicação da gestação com fisiopatologia ainda não completamente elucidada. A incidência na literatura é variável oscilando entre um caso para 8000 a 80000 partos $^{1-12}$. Devido a sua raridade foram criados registros nacionais nos Estados Unidos e Inglaterra, com critérios bastante estritos, o que provavelmente selecionou um subgrupo de maior gravidade $2,6,7,12,14$. Apesar de ter sido inicialmente descrita no Brasil em 1926, parece que os intensivistas e os obstetras brasileiros pouco conhecem desta entidade, refletindo na escassez de publicações nacionais ${ }^{5}$. O objetivo deste relato de caso foi chamar a atenção para este tipo de complicação e rever a literatura de maior relevância sobre a temática.

\section{RELATO DO CASO}

Paciente com 15 anos de idade, primigesta, 34 semanas de gestação com história de início de parto prematuro havia quatro dias, tendo sido internada em outro hospital onde fez uso de inibidores de trabalho de parto e dexametasona. Procurou o serviço de emergência do Hospital Monte Sinai na noite do dia em que recebera alta, com queixa de tosse e dispnéia de início pela manhã. Estava sem febre, lúcida, pressão arterial (PA) de 104/56 mmHg, pulso de 112 bpm, sem edema, pupilas isocóricas. Ao exame obstétrico sem anormalidades materno-fetais. Permaneceu em observação no setor de emergência onde se constatou rápido agravamento do quadro ventilatório, sendo transferida para a UTI. À admissão na unidade paciente apresentava-se lúcida, afebril, dispnéica (28 irm), taquicárdica (140 irm) e hipoxêmica $\left(\mathrm{SpO}_{2}\right.$ de $87 \%$ com máscara facial de reservatório e fluxo de $5 \mathrm{~L} / \mathrm{min}$ ), pressão arterial média de $64 \mathrm{mmHg}$. Com o agravamento do quadro ventilatório foi realizada intubação traqueal e submetida à ventilação mecânica modo pressão controlada e volume garantido em aparelho Evita quatro (Dragger, Alemanha) com $\mathrm{FiO}_{2}$ de $60 \%$, freqüência respiratória de 12 irm, volume-corrente (VC) de $500 \mathrm{~mL}$, Ti de 1s e PEEP de $10 \mathrm{cmH}_{2} \mathrm{O}$. Exames complementares evidenciaram infiltrado pulmonar bilateral; gasometria antes da intubação $\left(\mathrm{PaO}_{2}\right.$ de $62 \mathrm{mmHg}, \mathrm{PaCO}_{2}$ de $25 \mathrm{mmHg}$, BE de $-7,8$, bicarbonato padrão de $18 \mathrm{mmol} / \mathrm{L})$; leucograma (leucometria global de 16300, segmentados $87 \%$ e bastões 5\%); plaquetas de $152.000 / \mathrm{mm}^{3}$; TTPa $(34,7$ 
seg às 9 h00 e 57,4 às 18 h00 do mesmo dia, 43 e 30 seg no dia seguinte; com testemunho de 30 seg); PCR (65 mg/L, $45 \mathrm{mg} / \mathrm{L}$ e $6 \mathrm{mg} / \mathrm{L}$, respectivamente, nos dias subseqüentes; hemocultura (3 amostras negativas); ecocardiograma (sem anormalidades), ultra-sonografia obstétrica (feto sem anormalidades). Foi solicitada, pelo médico assistente, uma avaliação pela infectologia, que fez a hipótese de tratar-se de um caso de síndrome de desconforto respiratório agudo, secundária à sepse de foco pulmonar. Orientado o início do tratamento com antimicrobianos de amplo espectro por via venosa: vancomicina ( $1 \mathrm{~g})$ a cada 12 horas; imipenen $(1 \mathrm{~g})$ a cada 6 horas e ciprofloxacina $(400 \mathrm{mg})$ a cada 8 horas. Foi submetida à cesariana na manhã do dia da admissão. O diagnóstico foi revisto após discussão do caso com os médicos envolvidos, tendo em vista vários dados clínicos e de exames complementares contrários à etiologia infecciosa; tais como a ausência de febre, rápida deterioração do quadro clínico, a melhora radiológica significativa no dia seguinte da admissão e a apresentação clássica da síndrome satisfazendo os critérios de registro nacional norte americano e inglês. O recém-nascido recebeu índice de Apgar 1 e 8, porém com rápida recuperação após manobras pós-parto. $\mathrm{Na}$ UTI a paciente começou a apresentar hipotensão arterial, apesar da reanimação volêmica, exigindo uso de noradrenalina $(1300 \mu \mathrm{g} / \mathrm{h})$ por três dias. Extubada após três dias de ventilação mecânica; porém exigiu VMNI intermitente durante 48 horas. Realizada tomografia computadorizada de tórax no dia da alta para melhor documentação do caso.

Obteve alta da UTI após 6 dias e hospitalar após 13 dias da admissão totalmente assintomática

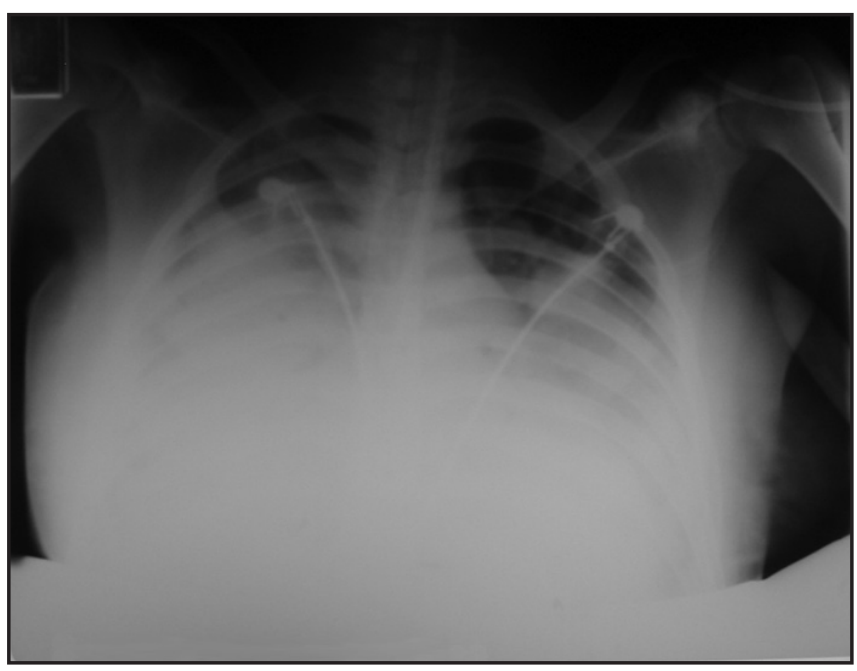

Figura 1 - Radiografia de Tórax na Admissão

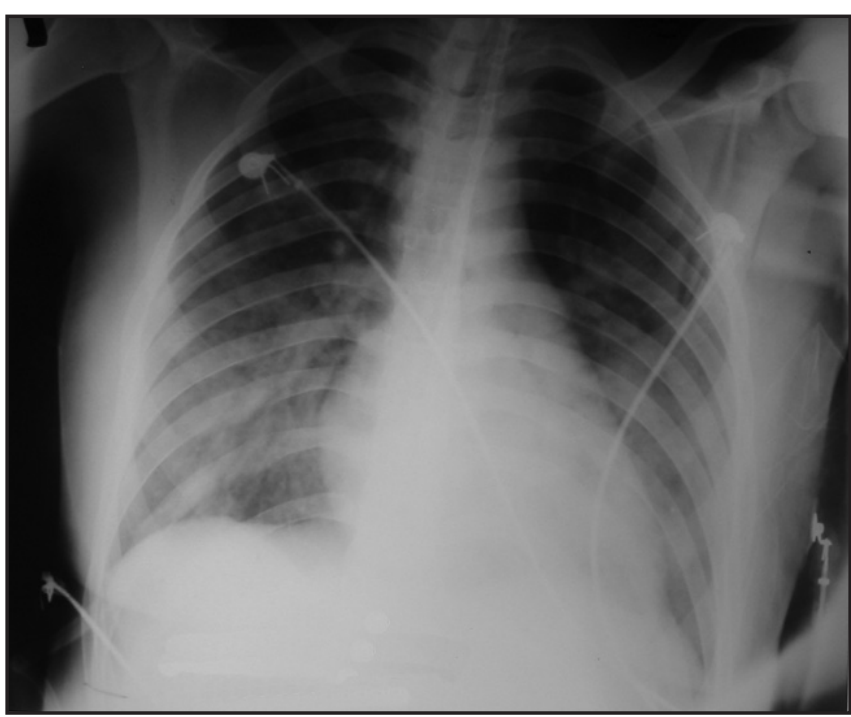

Figura 2 - Radiografia de Tórax na Alta

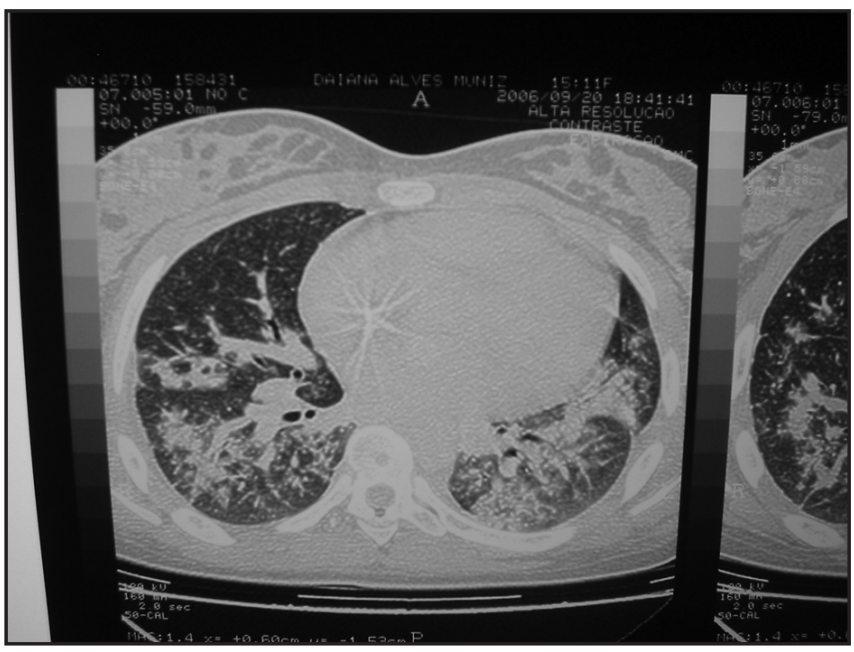

Figura 3 - Corte Tomográfico

\section{DISCUSSÃO}

A embolia por líquido amniótico é uma entidade clínica de incidência rara, em sua forma clássica, foi pela primeira vez relatada na literatura por Meyer em 1926, chefe do laboratório de Patologia da Faculdade de Medicina de São Paulo, porém somente após a publicação de Steiner em 1941 é que se tornou uma entidade clínica reconhecida ${ }^{1-5,10,12,13,17}$. A incidência em sua forma clássica é de 1 para cada 8.000 a 80000 partos; porém existem crescentes evidências de que a embolia por líquido amniótico apresenta amplo espectro de variação, devendo ter incidência bem maior e gravidade variável ${ }^{1-4,7,10,12-17}$. Neste hospital, com 12 anos de existência e 8533 partos, teve-se incidência no limite superior citado na literatura. Devido à sua raridade, 
pelo menos em sua forma clássica, a pesquisa sobre a sua fisiopatologia se concentrou em modelos animais heterogêneos, dificilmente extrapoláveis para seres humanos ${ }^{1-4,10,12,16}$. O melhor conhecimento da doença se deveu à criação de registros nacionais; inicialmente nos Estados Unidos e, posteriormente, na Inglaterra ${ }^{2,6}$. A fisiopatologia, ainda não completamente elucidada, se deve à passagem de elementos fetais particulados ou não para a circulação materna durante ou logo após o parto, aborto, amniocentese ou colocação de transdutor intra-uterino $3,4,10,12,15-17,22,24$. A presença destes elementos já foi detectada em muitos órgãos, porém a circulação pulmonar é o local de maior relevância. Como mecanismos fisiopatológicos faz-se necessário dividi-los em dois grandes grupos, mecânico e humoral. Em vários modelos experimentais e em algumas circunstâncias clínicas foi constatada uma oclusão vascular capaz de gerar repercussões funcionais significativas;não obstante na maioria das vezes o que se identifica é uma reação do organismo materno aos elementos fetais, gerando mediadores que seriam os responsáveis pelas repercussões orgânicas. Já foi documentada, do ponto vista experimental e algumas vezes clínico, a ativação da coagulação, da fibrinólise, da triptase, do complemento, da cascata do ácido araquidônico, da bradicinina, da endotelina e das citocinas ${ }^{1-}$ $5,9,10,12,16,20,21$. O quadro hemodinâmico parece se dever a depressão miocárdica reversível ás vezes associado à síndrome vasoplégica; enquanto o quadro respiratório parece ser inicialmente secundário à congestão pulmonar, sendo progressivamente substituído por um componente de edema pulmonar não cardiogênico devido à lesão pulmonar por mediadores. Os estudos com pacientes sempre evidenciaram coagulopatia, mesmo que presente só do ponto de vista laboratorial, caracterizada por parâmetros sugestivos de coagulação intravascular disseminada. Pouco se conhece sobre as manifestações em outros órgãos, que muitas vezes são secundárias ao quadro hemodinâmico e/ou discrásico. As manifestações cerebrais parecem ser, na maioria das vezes, secundárias à hipóxia'1-4,9,10,12,15,17,21,24,25.

Do ponto de vista clínico pode-se destacar três síndromes:

1. Síndrome hemodinâmica: caracterizada por parada cardíaca ou hipotensão arterial súbita;

2. Insuficiência respiratória;

3. Coagulopatia.

A apresentação clássica é de um quadro de insuficiência respiratória de início súbito, muitas vezes seguido de parada cardíaca. A coagulopatia geralmente surge do ponto de vista clínico ou laboratorial nas pacientes que sobrevivem ao evento catastrófico inicial. Não obstante qualquer das síndromes, mais raramente a coagulopatia, pode ser preponderante como sintomatologia inicial. A sintomatologia neurológica, tonteiras ou convulsões, muitas vezes é o sintoma de apresentação $1-4,8,10-12,16-20,22$.

O diagnóstico da embolia por líquido amniótico é de exclusão com outras afecções próprias da gestação ou que se descompensam durante a gravidez. Faz parte do diagnóstico diferencial a pré-eclâmpsia, eclâmpsia, sepse, miocardiopatia periparto, anafilaxia, tromboembolismo pulmonar, estenose mitral, entre outros. A pesquisa de elementos fetais na circulação pulmonar com material colhido através do cateter de artéria pulmonar apresenta várias limitações, desde a dificuldade de diferenciar os elementos fetais dos maternos na circulação pulmonar até a possibilidade de passagem do cateter em situações extremas, em que o tratamento inicial deve ser empírico. Existem alguns testes recentes, aparentemente promissores, de maior especificidade e de execução em sangue periférico; porém ainda estão em fase de pesquisa clínica ${ }^{1-4,10-13,23-25}$.

O tratamento é eminentemente de suporte, com destaque para a melhora das trocas gasosas pulmonares, suporte hemodinâmico com fármacos inotrópicos e vasopressores; e tratamento da coagulopatia,se necessário, com reposição dos fatores de coagulação ${ }^{1-4,10,12,15,17}$.

O caso relatado evidenciou quadros respiratórios, seguidos de instabilidade hemodinâmica e coagulopatia, somente presente no exame laboratorial. Houve respostas relativamente rápidas, permitindo a extubação da paciente no $3^{\circ}$ dia do início do quadro, com alta hospitalar assintomática; não obstante como substrato à provável mecanismo inflamatório pulmonar, ainda apresenta alterações no parênquima pulmonar na tomografia computadorizada.

\section{CONCLUSÃO}

A embolia por líquido amniótico é uma complicação rara da gestação que em sua forma clássica apresenta alta morbimortalidade materno-fetal; não obstante existem evidências crescentes de tratar-se de uma entidade com amplo espectro de gravidade e manifestações clínicas.

É de extrema importância o seu conhecimento, especialmente pelo intensivista e obstetra, com o intuito de reconhecimento precoce e manuseio adequado da gestante e do concepto. 


\section{REFERÊNCIAS}

01. Locksmith GJ - Amniotic fluid embolism. Obstet Gynecol Clin North Am, 1999;26:435-444.

02. Clark SL, Hankins GD, Dudley DA et al - Amniotic fluid embolism: analysis of the national registry. Am J Obstet Gynecol, 1995;172:1158-1169.

03. Moore J, Baldisseri MR - Amniotic fluid embolism. Crit Care Med, 2005;33:(Suppl10):S279-S285.

04. Davies $\mathrm{S}$ - Amniotic fluid embolism: a review of the literature. Can $\mathrm{J}$ Anaesth, 2001;48:88-98.

05. Meyer JR - Embolia pulmonar amnio caseosa. Bras Med, 1926;2:301303.

06. Tuffnell DJ - United kingdon amniotic fluid embolism register. BJOG. 2005;112:1625-1629.

07. Gilbert WM, Danielsen B - Amniotic fluid embolism: decreased mortality in a population-based study. Obstet Gynecol, 1999;93:973-977.

08. Bastien JL, Graves JR, Bailey S - Atypical presentation of amniotic fluid embolism. Anesth Analg, 1998;87:124-126.

09. Robillard J, Gauvin F, Molinaro G et al - The syndrome of amniotic fluid embolism: a potential contribution of bradykynin. Am J Obstet Gynecol, 2005;193:1508-1512.

10. Aurangzeb I, George L, Raoof S - Amniotic fluid embolism. Crit Care Clin, 2004;20:643-650.

11. Ray BK, Vallejo MC, Creinin MD et al - Amniotic fluid embolism with second trimester pregnancy termination: a case report. Can $\mathrm{J}$ Anesth, 2004;51:139-144.

12. Green BT, Umana E - Amniotic fluid embolism. South Med J, 2000;93:721 723.

13. Moore $\mathrm{J}$ - Amniotic fluid embolism: on the trail of an elusive diagnosis. Lancet, 2006;368:1399-1401.
14. Ratten GJ - Amniotic fluid embolism--2 case reports and a review of maternal deaths from this cause in Australia. Aust N Z J Obstet Gynaecol, 1988;28:33-35.

15. Schoening AM - Amniotic fluid embolism: historical perspectives \& new possibilities. MCN Am J Matern Child Nurs, 2006;31:78-83.

16. Marcus BJ, Collins KA, Harley RA - Ancillary studies in amniotic fluid embolism: a case report and review of the literature. Am J Forensic Med Pathol, 2005;26:92-95.

17. Fletcher SJ, Parr MJ - Amniotic fluid embolism: a case report and review. Resuscitation, 2000;43:141-146

18. Kramer MS, Rouleau J, Baskett TF et al - Amniotic-fluid embolism and medical induction of labour: a retrospective, population-based cohort study. Lancet, 2006;368:1444-1448.

19. Yang JI, Kim HS, Chang $\mathrm{KH}$ et al - Amniotic fluid embolism with isolated coagulopathy: a case report. J Reprod Med, 2006;51:64-66.

20. Malhotra P, Agarwal R, Awasthi A et al - Delayed presentation of amniotic fluid embolism: lessons from a case diagnosed at autopsy. Respirology, 2007;12:148-150.

21. Balazic J, Rott T, Jancigaj T et al - Amniotic fluid embolism with involvement of the brain, lungs, adrenal glands, and heart. Int J Legal Med, 2003;117:165-169.

22. Pluymakers $\mathrm{C}$, De Weerdt $\mathrm{A}$, Jacquemyn $\mathrm{Y}$ et al - Amniotic fluid embolism after surgical trauma: two case reports and review of the literature. Resuscitation, 2007;72:324-332.

23. Harboe T, Benson MD, Oi H et al - Cardiopulmonary distress during obstetrical anaesthesia: attempts to diagnose amniotic fluid embolism in a case series of anaphylaxis. Acta Anaesthesiol Scand, 2006;50:324-330.

24. James CF, Feinglass NG, Menke DM et al - Massive amniotic fluid embolism: diagnosis aided by emergency transesophageal echocardiography. Int J Obstet Anesth, 2004;13:279-283.

25. Huybrechts W, Jorens PG, Jacquemyn $Y$ et al - Amniotic fluid embolism: a rare cause of left-sided heart failure. Acta Cardiol, 2006;61:643-649. 